\title{
Small vessel disease disrupts EEG postural brain networks in 'unexplained dizziness in the elderly'
}

\author{
R.T. Ibitoye ${ }^{\mathrm{a}, \mathrm{b}}$, P. Castro ${ }^{\mathrm{a}}$, A. Desowska ${ }^{\mathrm{b}}$, J. Cooke ${ }^{\mathrm{a}}$, A.E. Edwards ${ }^{\mathrm{a}}$, O. Guven ${ }^{\mathrm{a}}$, Q. Arshad ${ }^{\mathrm{a}, \mathrm{c}}$, L. Murdin ${ }^{\mathrm{d}}$, \\ D. Kaski ${ }^{\text {a,e }}$, A.M. Bronstein ${ }^{\mathrm{a}, *}$ \\ a Neuro-otology Unit, Imperial College London, London, UK \\ ${ }^{\mathrm{b}}$ The Computational, Cognitive and Clinical Neuroimaging Laboratory (C3NL), Imperial College London, London, UK \\ ' inAmind Laboratory, Department of Neuroscience, Psychology and Behaviour, University of Leicester, Leicester, UK \\ ' Guy's and St Thomas' NHS Foundation Trust, London, UK \\ ${ }^{\mathrm{e}}$ Department of Clinical and Movement Neurosciences, University College London, London, UK
}

\section{A R T I C L E I N F O}

\section{Article history:}

Accepted 25 July 2021

Available online 30 August 2021

\section{Keywords:}

Small vessel disease

Dizziness

EEG

Elderly

Postural control

Unexplained dizziness in the elderly

\section{H I G H L I G H T S}

- Unexplained dizziness in the elderly may result from cerebral small vessel disease.

- Dizzy elderly patients differed from controls in EEG power when standing.

- EEG power when standing correlated with subjective (perceived) instability.

\begin{abstract}
A B S T R A C T
Objective: To examine the hypothesis that small vessel disease disrupts postural networks in older adults with unexplained dizziness in the elderly (UDE).

Methods: Simultaneous electroencephalography and postural sway measurements were undertaken in upright, eyes closed standing, and sitting postures (as baseline) in 19 younger adults, 33 older controls and 36 older patients with UDE. Older adults underwent magnetic resonance imaging to determine whole brain white matter hyperintensity volumes, a measure of small vessel disease. Linear regression was used to estimate the effect of instability on electroencephalographic power and connectivity. Results: Ageing increased theta and alpha desynchronisation on standing. In older controls, delta and gamma power increased, and theta and alpha power reduced with instability. Dizzy older patients had higher white matter hyperintensity volumes and more theta desynchronisation during periods of instability. White matter hyperintensity volume and delta power during periods of instability were correlated, positively in controls but negatively in dizzy older patients. Delta power correlated with subjective dizziness and instability.

Conclusions: Neural resource demands of postural control increase with age, particularly in patients with UDE, driven by small vessel disease.

Significance: EEG correlates of postural control saturate in older adults with UDE, offering a neurophysiological basis to this common syndrome.

(c) 2021 International Federation of Clinical Neurophysiology. Published by Elsevier B.V. This is an open access article under the CC BY-NC-ND license (http://creativecommons.org/licenses/by-nc-nd/4.0/).
\end{abstract}

\section{Introduction}

Cerebral small vessel disease is a common cause of covert vascular brain injury that contributes to age-related cognitive and balance decline (Wardlaw et al., 2019). Early effects of small vessel disease on networks mediating cognition are well understood (Veldsman et al., 2020), but implications for balance control and

\footnotetext{
* Corresponding author.

E-mail address: a.bronstein@imperial.ac.uk (A.M. Bronstein).
}

symptoms remain unclear. Unexplained dizziness in the elderly (UDE) lacks diagnostic criteria but its prevalence reaches $30 \%$ beyond 60 years of age, and $50 \%$ beyond 85 years (Jönsson et al., 2004). UDE may be a consequence of early small vessel disease (Ahmad et al., 2015; Fife and Baloh, 1993). A neurophysiological mechanism relating small vessel disease to postural symptoms, including dizziness, has however not been demonstrated.

The contribution of the cerebral cortex to postural control increases in ageing (Papegaaij et al., 2014) such that in older adults, balance increasingly depends on executive function (Boisgontier 
et al., 2013). Greater cortical activation across most cortical areas is seen with age during balance control and gait (Lin et al., 2017). In the prefrontal cortex, this ageing-related increase in activation likely compensates for declining neural efficiency (Nóbrega-Sousa et al., 2020; St George et al., 2021). Balance-related prefrontal activation may however decline in advanced age (Nóbrega-Sousa et al., 2020). Such a decline in activation has been suggested to reflect a saturation of compensatory mechanisms, consistent with established cognitive models of ageing (Cabeza, 2002; Park and Reuter-Lorenz, 2009). However, the relationship between pathophysiological processes in ageing and brain activity relevant to balance is not defined and, in this context, small vessel disease is likely to play a significant role.

EEG, being applicable during upright balance, informs neural mechanisms underpinning postural control. In young adults, as postural demands increase, delta and gamma power increase, whereas theta, alpha and beta power tend to decrease (Edwards et al., 2018; Hülsdünker et al., 2015; Ozdemir et al., 2018; Sipp et al., 2013; Slobounov et al., 2009). Delta oscillations linked to postural instability likely inhibit task-irrelevant networks (Harmony, 2013; Ozdemir et al., 2018). Theta, alpha and beta desynchronisation reflect executive, attentional and sensorimotor processes respectively (Edwards et al., 2018; Hülsdünker et al., 2015; Sipp et al., 2013; Slobounov et al., 2009). The effect of healthy ageing is unclear although a greater increase in gamma power in older adults compared to younger adults following postural challenge has been reported (Ozdemir et al., 2018).

We apply postural EEG in younger and older controls, hypothesising balance represents an increasingly demanding task with age (Edwards et al., 2018; Hülsdünker et al., 2015; Ozdemir et al., 2018; Sipp et al., 2013; Slobounov et al., 2009). We then combine EEG co-registered with postural sway, with automated white matter hyperintensity segmentation in older controls, to determine the effects of spontaneous instability on neural activity, and the influence of small vessel disease. We hypothesise that the burden of cerebral small vessel disease influences the cortical control of balance in older adults (i) as reflected by increased changes in EEG power in upright standing compared to seated rest, (ii) and by an increased influence of spontaneous sway on EEG power. We further hypothesise (iii) that idiopathic dizziness is characterised by a different mode of postural control, reflected in a different pattern of brain connectivity during standing balance.

\section{Methods}

\subsection{Participants}

Older adults with dizziness were recruited as part of a separate ongoing study. Forty-six dizzy participants were screened from neurology and neuro-otology clinics, and of these thirty-six (14 females, age $>60$ years, mean $77 \pm 7$ [standard deviation, SD] years) were recruited by the following criteria: (i) expert assessment found no relevant neurological, ophthalmological or vestibular diagnosis (thus they had UDE), (ii) peripheral vestibular function was normal (assessed by caloric testing, video head impulse test or rotating chair electronystagmography, excluding presbyvestibulopathy (Calic et al., 2020)) and (iii) clinical assessment and brain MRI identified no other neurological disease. None met diagnostic criteria for persistent postural-perceptual dizziness (PPPD), a functional vestibular disorder (Staab et al., 2017). Symptoms were long-standing (mean $6 \pm 5$ [SD] years) and no alternative diagnoses emerged following at least 6 months of follow-up. Another 33 non-dizzy older adults (14 females, age $>60$ years, mean $76 \pm 6$ [SD] years) were recruited as partners of patients, from a research register and from a local older persons group, following screening to exclude dizziness (all had a Dizziness Handicap Inventory score $=0$, no imbalance or spinning vertigo in the last 12 months, and no neurological disorders (Jacobson and Newman, 1990)). Video head impulse tests confirmed normal vestibular function (MacDougall et al., 2009). Mean horizontal video head impulse test gain was the measure of vestibular function. Written informed consent was obtained as approved by the local ethics research committee.

The burden of dizziness was quantified by the Vertigo Symptom Scale and the Dizziness Handicap Inventory (DHI) (Jacobson and Newman, 1990; Yardley et al., 1992). Fear of falling was assessed by the Falls Efficacy Scale (Tinetti et al., 1990). Premorbid intelligence was estimated by the National Adult Reading Test (NART) (Nelson, 1982). Reaction times were assessed by a decision task within the Cogstate ${ }^{\circledR}$ Brief Battery, a computerised cognitive assessment (Maruff et al., 2009). Vascular risk factors such as hypertension, diabetes, hypercholesterolaemia, obesity [body mass index $>30$ ], smoking [ $>20$ pack years] and previous transient ischaemic attacks were noted. Blood pressure and heart rate were assessed supine, immediately on standing and after 3 minutes of standing (as recommended by the European Society of Cardiology (Brignole et al., 2018)); mean changes between supine and standing measurements were the measures of orthostatic blood pressure change.

To determine the effect of healthy ageing on postural EEG, nineteen young adults aged 20-30 years were additionally recruited. They reported no dizziness or imbalance and had no known neurological or neuro-otological diagnoses.

\subsection{MRI acquisition and processing}

Structural MRI data were acquired on a Siemens 3-T Verio scanner in all older participants (Siemens Healthcare). Volumetric structural imaging was acquired using a T1-weighted MPRAGE sequence $(1-\mathrm{mm}$ thick transverse slices, repetition time $=2300 \mathrm{~m}$ $\mathrm{s}$, echo time $=2.98 \mathrm{~ms}$, flip angle $=9$, in-plane resolution $=1 \times 1 \mathrm{~m}$ $\mathrm{m}$, matrix size $=256 \times 256$, field of view $=25.6 \times 25.6 \mathrm{~cm})$. A fluidattenuated inversion recovery sequence (FLAIR) was used to identify white matter hyperintensities. Diffusion weighted images were also acquired (64 directions, $\mathrm{b}=1000 \mathrm{~s} / \mathrm{mm} 2$ with four interleaved $\mathrm{b}=0 \mathrm{~s} / \mathrm{mm} 2$, echo time $=103 \mathrm{~ms}$, repetition time $=9500 \mathrm{~ms}$, voxel size $2 \times 2 \times 2 \mathrm{~mm}$ ).

Voxel-wise white matter hyperintensity probabilities were determined by the Lesion Prediction Algorithm, a MATLAB ${ }^{\circledR}$ (R2019a, Natick, Massachusetts: The MathWorks Inc.) toolbox, validated in older populations (Schmidt et al., 2012). For white matter hyperintensity volume, a lesion probability threshold of $\mathrm{p}>.16$ was used; this value has been shown to be optimal in the context of small vessel disease (Guerrero et al., 2018). Images were registered to a common (Montreal Neurological Institute) template prior to whole-brain volume calculations, to account for interindividual variability in intracranial volume.

\section{3. $E E G$}

\subsubsection{Acquisition}

Each participant underwent 32 channel EEG recording (Waveguard $^{\mathrm{TM}}$ cap, ANT Neuro, Enschede, The Netherlands) at 1250 samples per second with eyes closed in both sitting (high backed chair) and standing postures (with shoes off at comfortable stance width, so subjective comfort was not a confound, Fig. 1A). Five 2minute recordings were undertaken in each condition, with a brief seated rest interval, alternating between conditions, giving a total of 20 minutes of EEG. Immediately after each standing recording, 


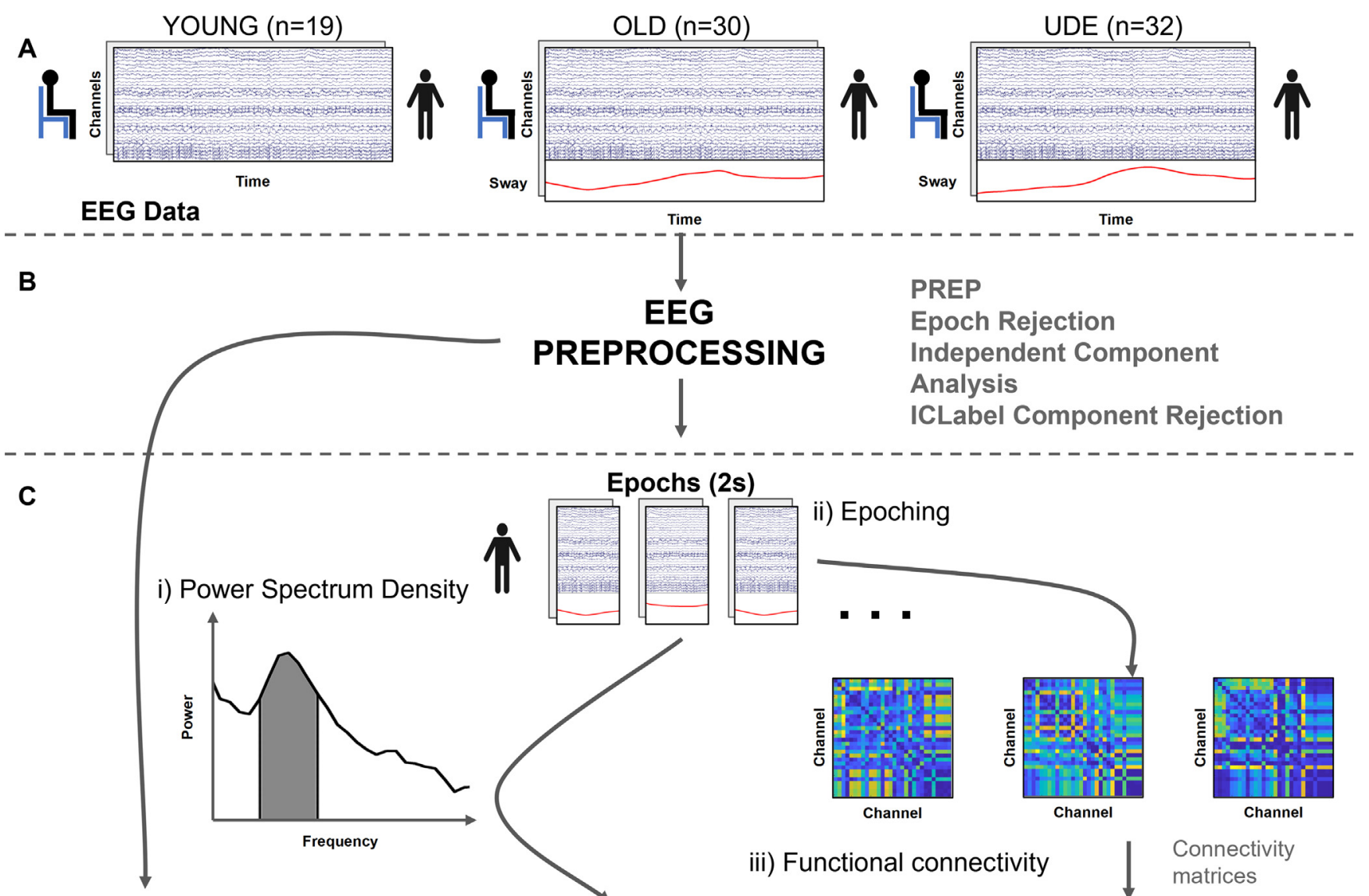

D i) Normalised power

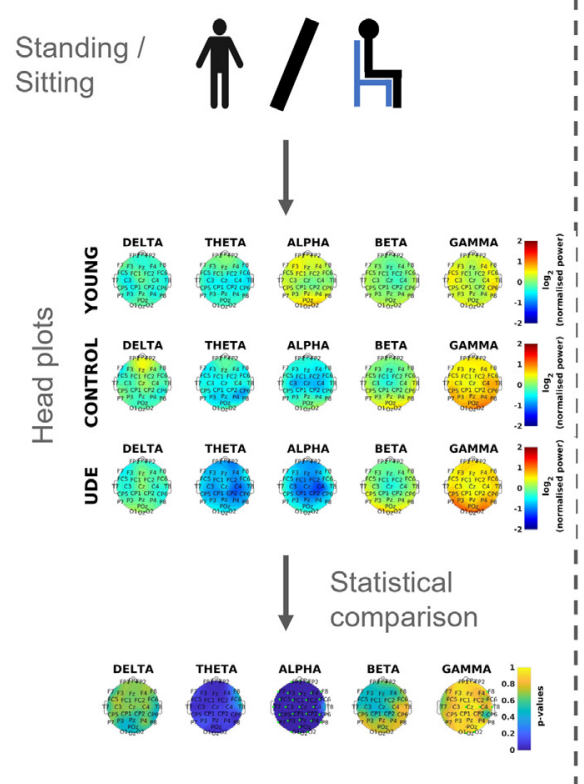

D ii) Power-sway relationship

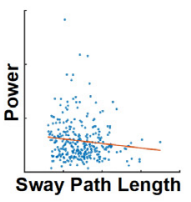

Coefficient of
regression line
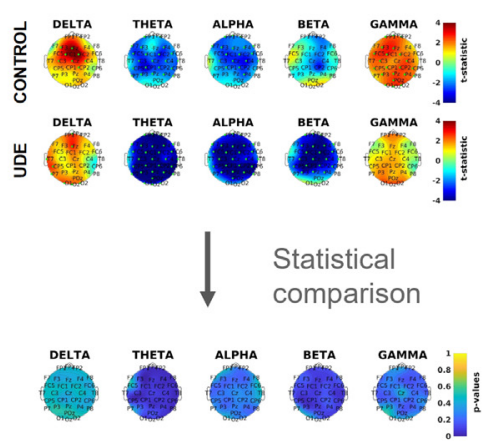

D iii) Connectivity-sway relationship

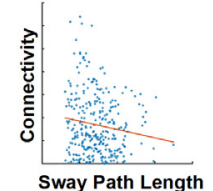

Coefficient of regression line

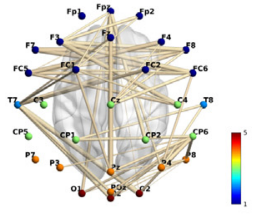

Network Based Statistics

E) Further Analysis

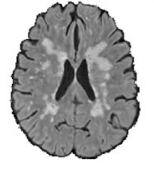

Correlation with

MRI data

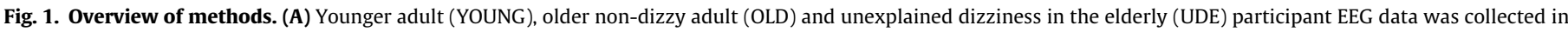

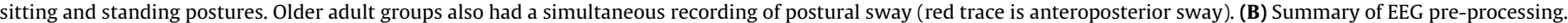

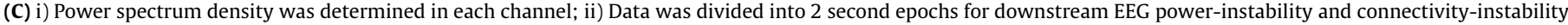

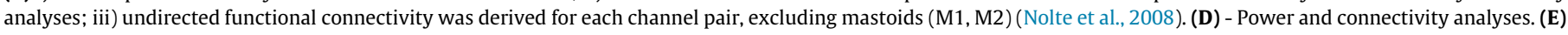
- Further analysis investigated the influence of white matter hyperintensity volume on power-instability, and connectivity-instability findings.

participants were asked to rate their instability on a scale between 0 and 10 (Castro et al., 2019). Three datasets from the non-dizzy older group, and four datasets from the dizzy group were excluded because of poor recording quality.
An electromagnetic tracking device (Fastrak; Polhemus, USA) was firmly taped over the occiput and recorded antero-posterior linear head displacement in space (sway, $\mathrm{mm}$ ) in the standing condition. The signal was digitised using a custom-built digital-to- 
analogue converter. This signal was then connected to an additional channel on the ANT ${ }^{\circledR}$ Neuro amplifier, co-registering sway and EEG data.

\subsubsection{Preprocessing}

EEG data was processed within EEGLAB $^{\circledR}$ (see below, Fig. 1B) (Delorme and Makeig, 2004). Raw EEG data were preprocessed using the PREP pipeline which incorporates $50 \mathrm{~Hz}$ line noise removal using CleanLine (an adaptive filter using frequencydomain multi-taper regression to remove sinusoidal artefacts) and a $1 \mathrm{~Hz}$ zero-phase high pass filter (Hamming windowed sinc finite impulse response), a $100 \mathrm{~Hz}$ zero-phase low-pass 4 th order Butterworth filter, epoch-based rejection using frequency and joint probability criteria, subject-level concatenated independent component analysis (ICA) and automated component classification using ICLabel (Fig. 1B) (Bigdely-Shamlo et al., 2015; Makeig et al., 2004; Pion-Tonachini et al., 2019). Identified components were also visually inspected prior to rejection.

\section{Analysis}

\subsection{General}

Frequency bands in EEG were defined as follows: delta: 1-4 Hz, theta $4-8 \mathrm{~Hz}$, alpha $8-14 \mathrm{~Hz}$, beta $14-30 \mathrm{~Hz}$ and gamma $30-$ $100 \mathrm{~Hz}$. Group comparisons were undertaken using contrasts within multiple linear regression models. To adjust for multiple comparisons across 32 channels while remaining sensitive to spatially contiguous activation, we applied threshold-free cluster enhancement (TFCE) and non-parametric permutation testing (10,000 iterations) (Mensen and Khatami, 2013; Smith and Nichols, 2009).

\subsection{Power spectrum density}

For each channel, mean power spectrum density (PSD, microvolts ${ }^{2} / \mathrm{Hz}$ ) was quantified using Welch's estimator as implemented in EEGLAB (Fig. 1C) (Delorme and Makeig, 2004). 'Normalised power', defined as standing power (PSD) divided by sitting power (PSD), was determined, reducing the effect of inter-individual variability in EEG power (Edwards et al., 2018).

\subsection{Connectivity}

Functional connectivity between channels, within each frequency band, was determined using the bivariate phase slope index (implemented in FieldTrip (Nolte et al., 2008; Oostenveld et al., 2011)). Phase slope index estimates coupling between source signals, and is robust to noise (Nolte et al., 2008). We used absolute phase slope index values as measures of undirected connectivity.

\subsection{Epoching}

To determine the relationship between EEG power or connectivity, and sway, we produced 2 second epochs from upright EEG data in a sliding window with 100 millisecond steps. This generated a per-participant mean of 4880 epochs across older controls (standard deviation 472), and 4848 epochs across participants with unexplained dizziness in the elderly (standard deviation 596); the two groups did not significantly differ in the number of epochs processed $(t(60)=0.224, p=.82)$. For each epoch, sway path length, and power and functional connectivity (phase slope index) in each frequency band, were determined (Hufschmidt et al., 1980; Nolte et al., 2008). Using linear regression, the coefficients of power, and separately, connectivity as predicted by sway magnitude (as a measure of instability) were derived. These produced participant and channel-specific measures of power-instability and connectivity-instability relationships whose summary statistics were used in higher order models (Beckmann et al., 2003) (Fig. 1D).

\subsection{Threshold-free cluster enhancement}

Permutation tests are an established way to conduct statistical testing while adjusting for the effects of multiple comparisons/hypothesis tests (Nichols and Holmes, 2002). A strength of permutation testing is the provision for exact control over the rate of false discovery across multiple hypothesis tests - the family-wise error rate. This control is achieved while being robust to the statistical distribution of the underlying data. A key principle underlying permutation testing is exchangeability - that for a specific null hypothesis, rearrangements of the data have no effect on a statistic of interest. The experimental question is often modelled using multiple linear regression (Winkler et al., 2014). Techniques have been developed for efficient and accurate permutation within linear models (Freedman and Lane, 1983).

In neuroimaging, multiple data points with a defined spatial relationship are often generated (e.g. channels in EEG, voxels in MRI). Conventional approaches to data analysis typically fit one data point at a time. The testing of multiple hypotheses (one for each data point) however necessitates subsequent correction of derived statistics to control the family-wise error rate. A number of approaches to this problem have been developed. These take into account the spatial structure of the data points and are sensitive to spatially clustered signals (Bansal and Peterson, 2018). Conventional cluster-based permutation tests require two arbitrary thresholds which may influence results: (i) a threshold for the inclusion of voxels within a cluster, and (ii) a threshold for cluster significance. Threshold-free cluster enhancement (TFCE) was developed to address the limitations of arbitrary threshold decisions (Smith and Nichols, 2009), by integrating the evidence for clustering across thresholds. A further strength of TFCE is the determination of statistical significance at the level of individual data points, rather than clusters. Though initially developed for the analysis of MRI data, TFCE has since been developed for EEG analyses in channel/source space (Mensen and Khatami, 2013).

Although 'threshold-free', TFCE requires two parameters to be defined which weigh the relative influences of spatial extent (E) and the degree of 'activation' $(H)$ in the integration of evidence across thresholds. Empirical and random field theory justifications have been provided for values of $\mathrm{E}=2 / 3$ and $\mathrm{H}=2$ (Smith and Nichols, 2009), and these values have also been shown to be appropriate for EEG data (Mensen and Khatami, 2013). A family-wise error rate $\mathrm{p}<.05$ within each frequency band was defined as statistically significant.

\subsection{Linear regression and cortical postural control}

Spontaneous instability likely drives intermittent neural responses in the control of posture (Loram et al., 2011). The relationship between cortical activity while standing (e.g. increases and decreases in power or connectivity) and sway thus provides insight into cortical processing relevant to the control of posture. Power (mean PSD in microvolts ${ }^{2} / \mathrm{Hz}$ using Welch's estimator (Delorme and Makeig, 2004)) was estimated for each epoch, channel ( $n=30$; i.e. the two mastoid channels were excluded from analysis) and frequency band $(n=5)$. Connectivity (bivariate phase slope index (Nolte et al., 2008)) was estimated for each of 435 connections $\left(n \frac{(n-1)}{2}=\frac{30 \times 29}{2}=435\right)$. We looked for regression evidence of a linear relationship between sway (the independent variable) and our EEG measures of interest: power and connectivity (depen- 
dent variable[s] in separate regressions). As regression coefficients are known to be sub-optimal for permutation testing in neuroimaging (Winkler et al., 2014), the t-statistic of these coefficients was instead used as an input to permutation testing and TFCE (Mensen and Khatami, 2013; Winkler et al., 2014).

\subsection{Network based statistics}

To test whether connectivity-instability measures were significant (i.e. differed from zero), we used the Network Based Statistic toolbox (Zalesky et al., 2010). Network Based Statistics combines general linear models with permutation tests to undertake statistical tests of connectivity in network space (Zalesky et al., 2010). The method searches for contiguous networks maximising a statistic (in our case, intensity, the sum of supra-threshold connection strengths) against a null distribution defined by permutation $(10,000$ iterations, Fig. 1D). The threshold for the inclusion of connections within networks was set at $\mathrm{p}<.05$. To identify significant EEG connectivity-postural instability relationships at group level, within-group one sample t-tests were undertaken by permutation within Network Based Statistics. Within each band, a network family-wise error rate $\mathrm{p}<.05$ was deemed statistically significant.

\section{Results}

4.1. Healthy ageing is associated with greater theta and alpha desynchronisation, and increased gamma power on standing

We determined the effect of healthy ageing on average EEG power changes on standing (normalised power $=$ PSD (standing) / PSD(sitting)), by comparing older controls to younger adults, postulating changes consistent with greater postural task difficulty (delta and gamma power increases, and theta, alpha and beta desynchronisation) (Edwards et al., 2018; Hülsdünker et al., 2015; Ozdemir et al., 2018; Sipp et al., 2013; Slobounov et al., 2009). We derived normalised power across frequency bands in both groups.

On standing, central theta and beta power decreased, and alpha and gamma power increased in peri-central areas in younger adults (Fig. 2A). In older adults normalised power increased frontally in delta, occipitally in beta, and globally in gamma; normalised power decreased centro-parietally in theta, and centrally in alpha (Fig. 2A). Statistical comparison between groups revealed older adults had significantly lower normalised theta and alpha power, and higher normalised gamma power compared to younger adults (Fig. 2B).

4.2. Instability drives frontal delta and gamma oscillations, and theta and alpha desynchronisation in healthy older adults

We investigated if spontaneous instability drove delta and gamma oscillations, as well as theta, alpha and beta desynchronisation in older adult controls, consistent with known EEG correlates of postural challenge (Edwards et al., 2018; Hülsdünker et al., 2015; Ozdemir et al., 2018; Sipp et al., 2013; Slobounov et al., 2009). We therefore determined the linear relationship between upright (standing) EEG power (PSD) and postural sway (instability) across epochs of EEG data. More sway correlated significantly with more delta power in frontocentral areas and more gamma power globally (Fig. 3C). More sway correlated significantly with less theta and alpha power in central and paracentral areas (Fig. 3C).
4.3. White matter hyperintensity volume correlates with greater delta power during periods of instability in healthy older adults

We investigated whether white matter hyperintensity volume correlated with the EEG power-instability coefficient (the linear relationship between power in the standing condition and sway), to suggest small vessel disease modulates the scaling of neural responses to instability. White matter hyperintensity volume correlated positively with the power-instability coefficient only in the delta frequency band, such that greater white matter hyperintensity volume was associated with greater delta power increases in periods of instability (Fig. 4A).

4.4. Normal vestibular function and postural performance but greater subjective instability in UDE

Vertigo symptom scale scores, a measure of overall dizziness, were moderately high in patients (median 17, Table 1) and they reported moderate dizziness-related handicap (DHI median 34). Falls efficacy scale scores, a measure of fear of falling, were significantly higher than in controls (Table 1 ).

We assessed the degree of postural sway while standing to determine whether participants with UDE were more unsteady than older controls. Neither sway path length, nor the root mean square of sway differed significantly between groups (Table 1 ).

Despite equivalent postural sway levels, the UDE group reported significantly greater instability than older controls (Subjective Instability Rating, Table 1). Orthostatic blood pressure did not differ significantly between groups, either immediately following standing, or after 3 minutes (Table 1). Video head impulse test gain, a measure of vestibular function, did not differ between UDE and older controls.

\subsection{More vascular risk factors and lower premorbid intelligence in unexplained dizziness in the elderly}

We quantified vascular risk factor burden, given its relevance to small vessel disease, and white matter hyperintensity volume as a marker of small vessel disease (Wardlaw et al., 2013). Participants with UDE had significantly higher total vascular risk burdens than older controls (Table 1), and a tendency to higher white matter hyperintensity volumes (Table 1 ). Hypertension and hypercholesterolaemia were significantly more prevalent in UDE (Table 1).

We assessed reaction time as a measure of central processing speed, and vocabulary as a measure of premorbid intelligence. Reaction time (detection task) was significantly longer in UDE than in older controls (Table 1; not significant after correction for multiple comparisons). Premorbid intelligence (National Adult Reading Test), was significantly lower in UDE than in older controls (Table 1).

\subsection{Greater instability-related theta desynchronisation in unexplained dizziness in the elderly}

We compared normalised power (PSD(standing) / PSD(sitting)) in UDE patients to older controls to investigate whether UDE was associated with excess of ageing-associated changes in EEG power on standing - delta and gamma power increases, and theta, alpha and beta power decreases (Edwards et al., 2018; Hülsdünker et al., 2015; Ozdemir et al., 2018; Sipp et al., 2013; Slobounov et al., 2009). Central theta and alpha power decreased and gamma power increased in UDE (Fig. 3A) on standing (relative to sitting). Statistical comparison of older controls and UDE using TFCE showed normalised theta and alpha power were significantly lower in UDE compared to older controls (Fig. 3B). 
A

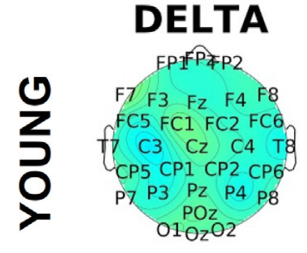

\section{DELTA}

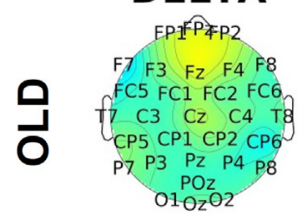

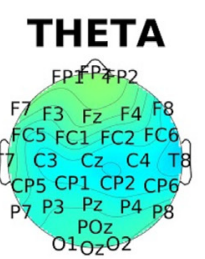
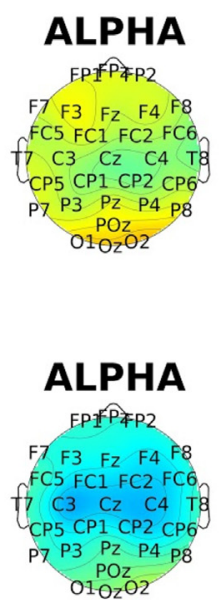
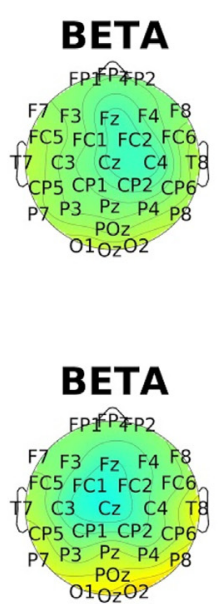
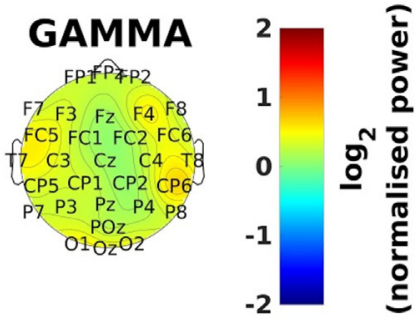

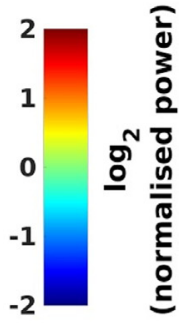

B

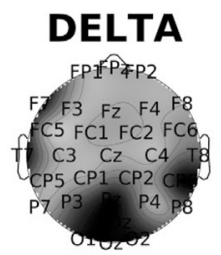

OLD > YOUNG
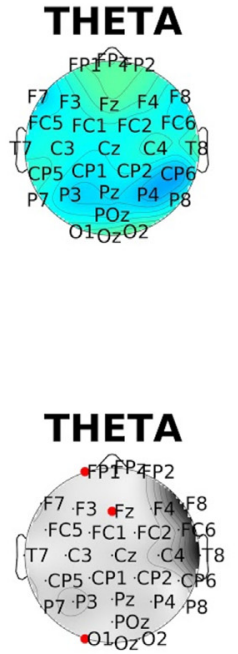

YOUNG > OLD

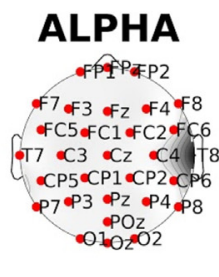

YOUNG > OLD

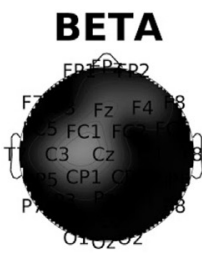

YOUNG > OLD
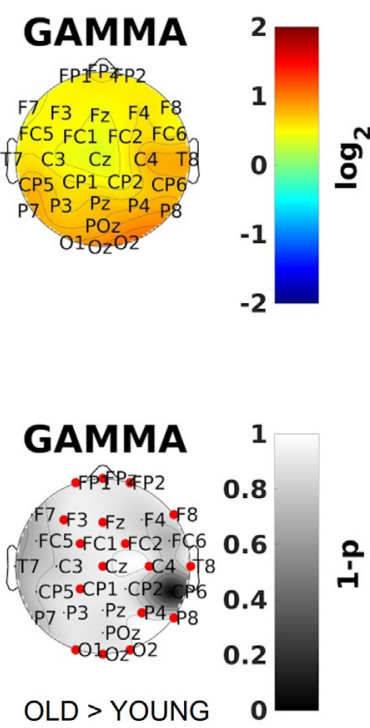

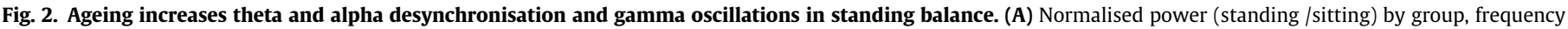

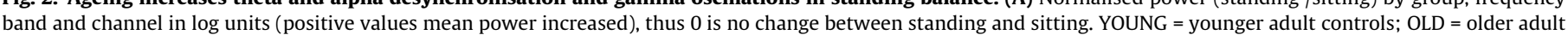

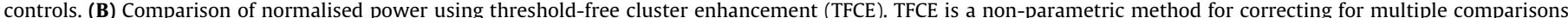

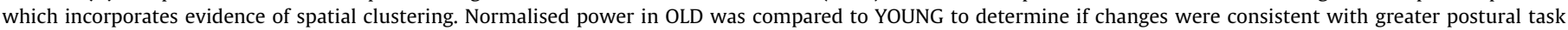

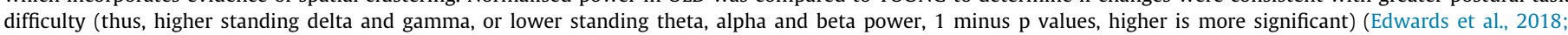

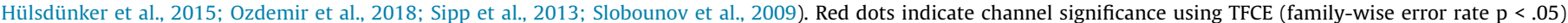
(Smith and Nichols, 2009).

A
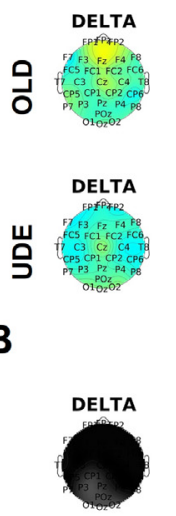

UDE > OLD
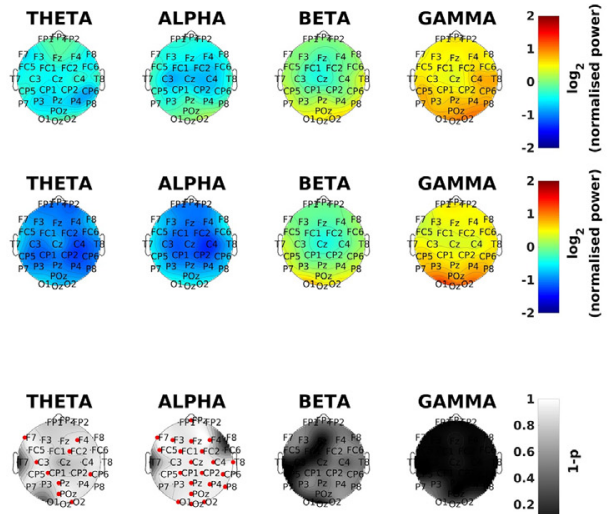

$\mathrm{OLD}>\mathrm{UDE}$
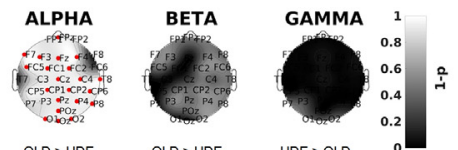

C
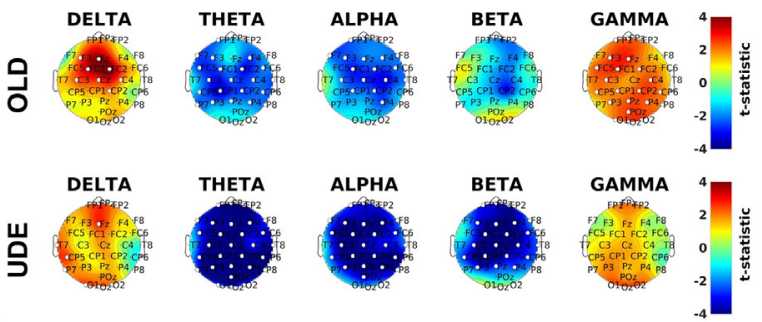

D
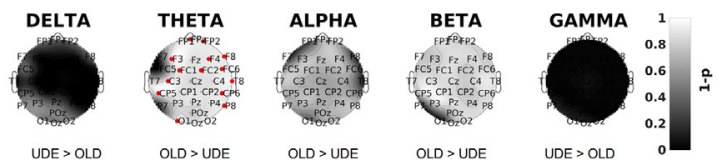

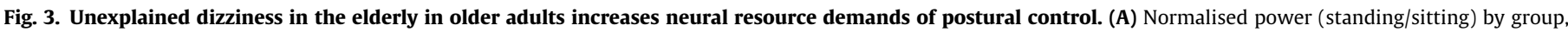

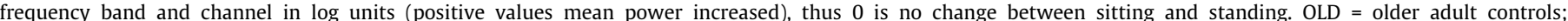

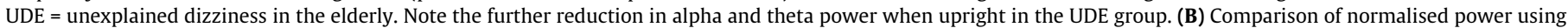

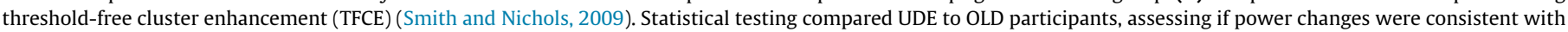

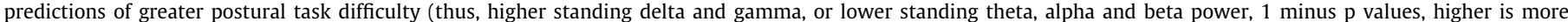

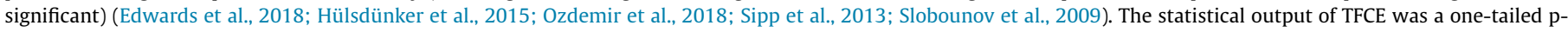

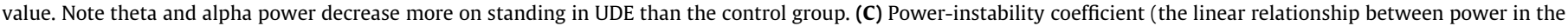

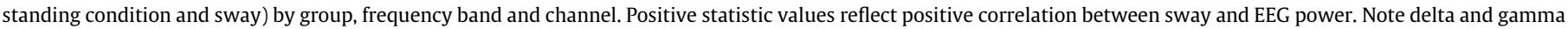

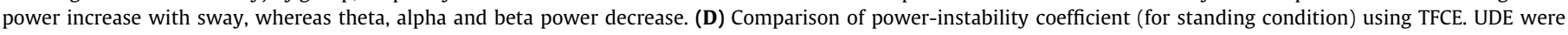

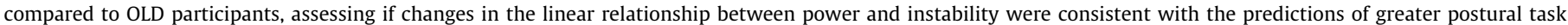

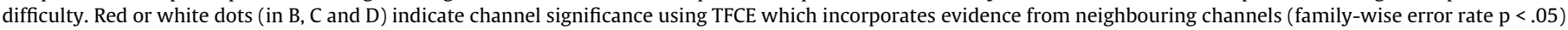
(Smith and Nichols, 2009). Note theta power decreases more with sway in UDE than the control group. 
A
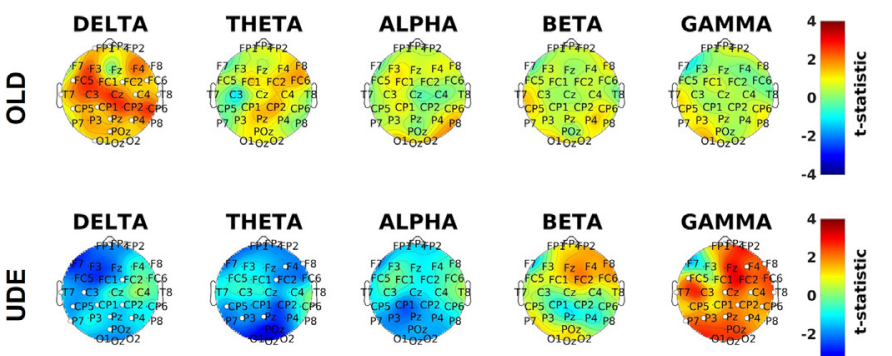

B
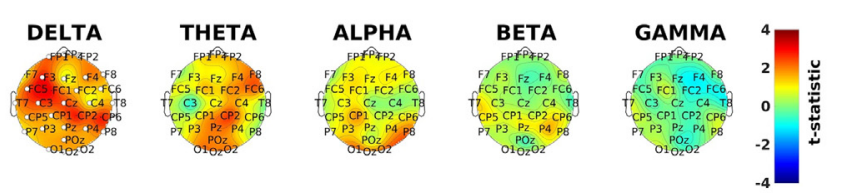
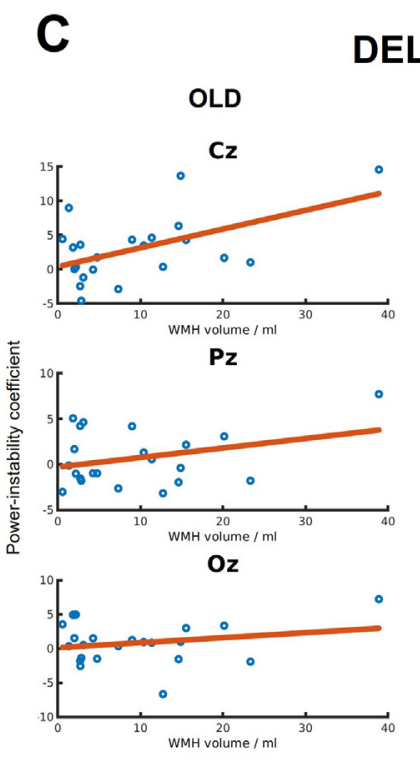

\section{DELTA}

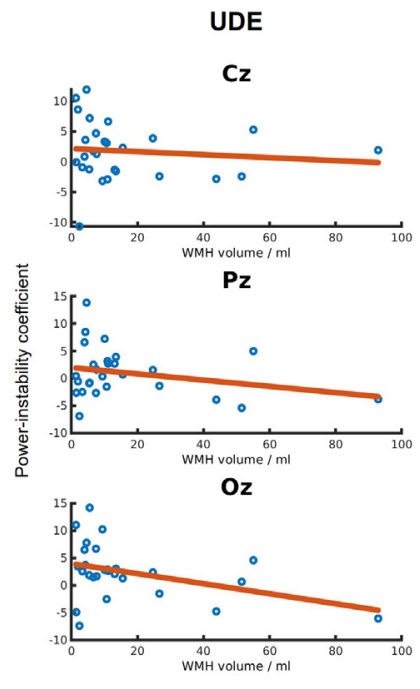

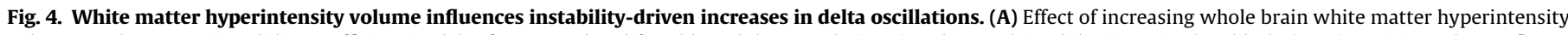

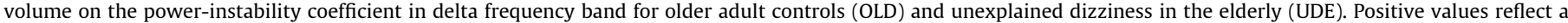

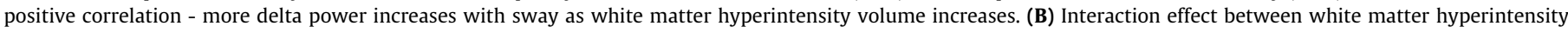

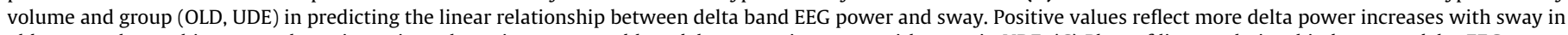

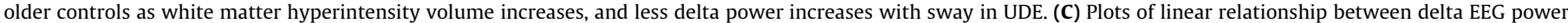

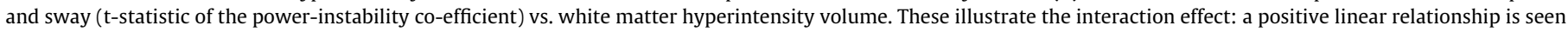

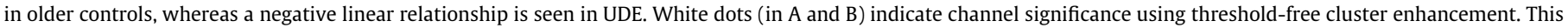
significance takes into account the spatial evidence of clustering of correlations.

We then investigated whether UDE patients had greater instability (sway)-driven changes in EEG power than older controls in keeping with standing being more challenging for UDE patients than older controls (greater delta and gamma, lower theta, alpha and beta power) (Edwards et al., 2018; Hülsdünker et al., 2015; Ozdemir et al., 2018; Sipp et al., 2013; Slobounov et al., 2009). In UDE patients, more sway correlated with more delta power; less sway correlated significantly with more theta, alpha and beta power (correlation statistics in Fig. 3C). UDE patients were compared to older controls using TFCE. This showed the linear relationship between power and sway was significantly weaker in UDE than in controls in the theta frequency (Fig. 3D). The linear relationship between EEG power and body sway did not differ significantly between the two groups in delta or gamma frequency bands (Fig. 3D).

\subsection{White matter hyperintensity volume correlates with less delta power during periods of instability in unexplained dizziness in the elderly}

We investigated whether total (whole brain) white matter hyperintensity volume correlated positively with the powerinstability coefficient (the linear relationship between power in the standing condition and sway) in UDE patients as observed in controls. In UDE, total white matter hyperintensity volume had a negative correlation with the power-instability coefficient within delta, such that greater white matter hyperintensity volume was associated with significantly less increase in delta power in periods of instability (Fig. 4A). Additionally, in theta, white matter hyperintensity volume had a significant negative correlation with powerinstability coefficients (Fig. 4A). In gamma, white matter hyperintensity volume had a significant positive correlation with powerinstability coefficients (Fig. 4A).

In view of the apparent dissociation between groups in delta, we tested for interaction between group (UDE/older controls) and white matter hyperintensity volume in the prediction of power-instability coefficients. Group significantly moderated the effect of white matter hyperintensity volume on the prediction of power-instability coefficients only within the delta band (Fig. 4B and Fig. 4C).

\subsection{Different postural control networks during upright stance in unexplained dizziness in the elderly compared to controls}

We investigated postural-EEG connectivity to determine if networks underpinning balance in dizzy patients compared to controls were different or modified, indicating an altered mode of postural control. We thus determined connectivity as predicted by sway (instability) using linear regression (connectivity = depen dent variable, sway = dependent variable). Network Based Statistics was used to determine networks of significant positive, or negative correlation between connectivity and instability within each group. Significant theta, alpha and beta instability-sensitive networks were identified in controls (Fig. 5), whereas significant delta and gamma networks were found in UDE (Fig. 5). In older controls, both the theta and alpha instability-sensitive networks had negative connectivity-instability coefficients, thus connectivity decreased with increasing sway. The beta network had positive connectivity-instability coefficients, so connectivity increased with increasing sway. In UDE, the identified instability-sensitive delta frequency band network had positive connectivity-instability coefficients whereas the gamma network had negative connectivityinstability coefficients.

Thus for each frequency band, a statistically significant network wherein connectivity varied with sway was found in only one or the other of the two groups (older controls or UDE). Thresholding for significance is however an inherently arbitrary decision. Nonsignificant results may thus have masked networks just below the threshold for significance. To allow clearer visualisation of patterns of postural-EEG connectivity in patients and controls, within each frequency band and for each significant network, we identified the largest non-significant network in the other group. This 
Table 1

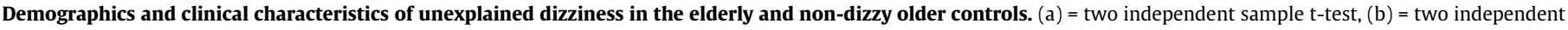

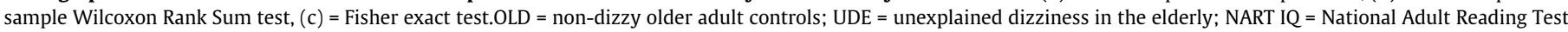

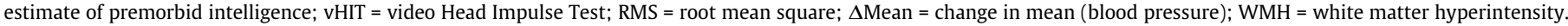

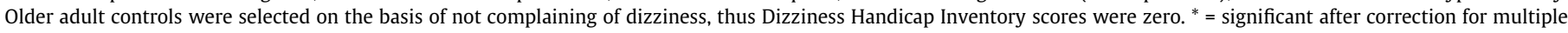
comparisons by False Discovery Rate (Genovese et al., 2002).

\begin{tabular}{|c|c|c|c|}
\hline & OLD & UDE & $\mathbf{p}$ \\
\hline$n$ & 33 & 36 & - \\
\hline Age & $76(6.0)$ & $77(6.5)$ & $0.41(a)$ \\
\hline Sex & $13 \mathrm{~F}$ & $14 \mathrm{~F}$ & - \\
\hline With Vascular Risk Factors & 19 & 31 & $0.015^{*}(\mathrm{c})$ \\
\hline Heart Disease (n) & 4 & 9 & $0.23(\mathrm{c})$ \\
\hline Hypertension (n) & 8 & 25 & $<0.001^{*}(\mathrm{c})$ \\
\hline Diabetes $(n)$ & 2 & 7 & $0.16(\mathrm{c})$ \\
\hline Hypercholesterolaemia ( $n$ ) & 6 & 23 & $<0.001^{*}(\mathrm{c})$ \\
\hline Obesity (n) & 0 & 5 & $0.055(\mathrm{c})$ \\
\hline Smoking - pack years (n) & 5 & 7 & $0.75(\mathrm{c})$ \\
\hline Previous TIA $(n)$ & 1 & 3 & $0.62(\mathrm{c})$ \\
\hline Subjective Instability Rating (Castro et al., 2019) & $1.2(1.6)$ & $3.5(3.6)$ & $<0.001^{*}(\mathrm{~b})$ \\
\hline Dizziness Handicap Inventory & $0(0)$ & $34(23)$ & $<0.001^{*}(\mathrm{~b})$ \\
\hline Vertigo Symptom Scale & $0(1)$ & $17(12)$ & $<0.001^{*}(\mathrm{~b})$ \\
\hline Falls Efficacy Scale & $7(1)$ & $13(5)$ & $<0.001^{*}(\mathrm{~b})$ \\
\hline NART IQ & $120(4)$ & $115(10)$ & $0.007^{*}(\mathrm{~b})$ \\
\hline Detection (reaction time) / $\log [s]$ ) & $2.54(0.107)$ & $2.59(0.0891)$ & $0.043(\mathrm{~b})$ \\
\hline vHIT gain (mean horizontal) & $1.0(0.13)$ & $1.0(0.23)$ & $0.84(\mathrm{~b})$ \\
\hline Normalised Total Sway Path Length / mm s $\mathrm{s}^{-1}$ & $7.6(2.3)$ & $8.8(4.6)$ & 0.19 (b) \\
\hline Root Mean Square of Sway / mm (Prieto et al., 1996) & $5.3(1.6)$ & $5.4(2.8)$ & $0.60(b)$ \\
\hline \multicolumn{4}{|l|}{$\begin{array}{l}\text { Blood Pressure [Systolic / Diastolic] / mmHg } \\
\quad \text { Supine }\end{array}$} \\
\hline$\triangle$ Mean, Immediate Standing & $151 / 75(17 / 9)$ & $154 / 76(17 / 10)$ & $0.48 / 0.63(a)$ \\
\hline \multirow{2}{*}{$\Delta M e a n, 3$ minute Standing } & $-2.0(7.8)$ & $-2.6(14)$ & $0.84(a)$ \\
\hline & $-3.8(21)$ & $2.8(13)$ & $0.13(a)$ \\
\hline WMH volume / ml & $5.7(10)$ & $7.8(17)$ & $0.24(\mathrm{~b})$ \\
\hline
\end{tabular}

non-significant network had the same direction of correlation (positive or negative relationship) between connectivity and sway in both groups. The identified non-significant networks and their p-values are shown in Fig. 5. None of the non-significant networks bordered on statistical significance (i.e. $\mathrm{p}>.1$ for all non-significant networks). In all cases the non-significant network also had fewer connections than its significant counterpart (the significant network for the other group, in the same frequency band). These findings imply the two groups (older controls and UDE) differed in the EEG connectivity networks engaged during standing balance.

\subsection{Frontal and centro-parietal delta power correlates with subjective instability}

Given the relevance of delta oscillations to executive control and postural responses (Harmony, 2013; Ozdemir et al., 2018), we investigated whether delta power when subjects were upright (standing) correlated positively with subjective instability in the delta frequency band. Across all older adults, delta power (PSD) while standing correlated significantly with subjective instability in frontal and centro-parietal areas (Fig. 6). Post-hoc tests within each group confirmed correlation in older controls but not in UDE (Fig. 6). Of note, seated delta power did not correlate with subjective instability.

\subsection{EEG preprocessing}

As EEG pre-processing, particularly ICA, could bias results by removing unequal amounts of data from each group, we compared the removal of components between groups; there were no significant differences. Specifically, younger controls did not differ significantly from older controls in the number of ICA components rejected (median 8 [IQR 5] vs. median 9 [IQR 4], p = .11). UDE and older controls also did not differ significantly in the number of ICA components rejected (median 9.5 [IQR 6] vs. median 9 [IQR 4], p = .99).

\section{Discussion}

Upright 'dizziness', often referred to as 'unexplained dizziness in the elderly', is a common neurological symptom in older adults that remains idiopathic in a large proportion of patients, and represents a clinical challenge for general practitioners, geriatricians, otologists and neurologists (Ahmad et al., 2015; Colledge et al., 1994). While dizziness has been associated with small vessel disease (separate to the established association of small vessel disease with manifest gait disturbance), its neural basis has not previously been investigated (Cerchiai et al., 2017). We investigated the effects of small vessel disease on the cortical control of balance in dizzy and control participants using sensitive neurophysiological methods applicable in a quiet, unperturbed, albeit challenging (eyes closed) upright posture. Our results showed ageing increased the neural resource demands of postural control. We found small vessel disease was a key factor in these results, increasing delta oscillations that likely drive a compensatory top-down executive control strategy (Harmony, 2013). This suggests that even in asymptomatic older adults, small vessel disease has measurable subclinical effects on cortical resource demands during postural control. The finding of even greater neural resource demands in UDE confirms deficits in cortical postural control are core to this syndrome. The engagement of different postural networks in dizzy patients compared to controls is compatible with a shift in the mode of postural control in patients. Our finding of correlation 


\section{OLD}
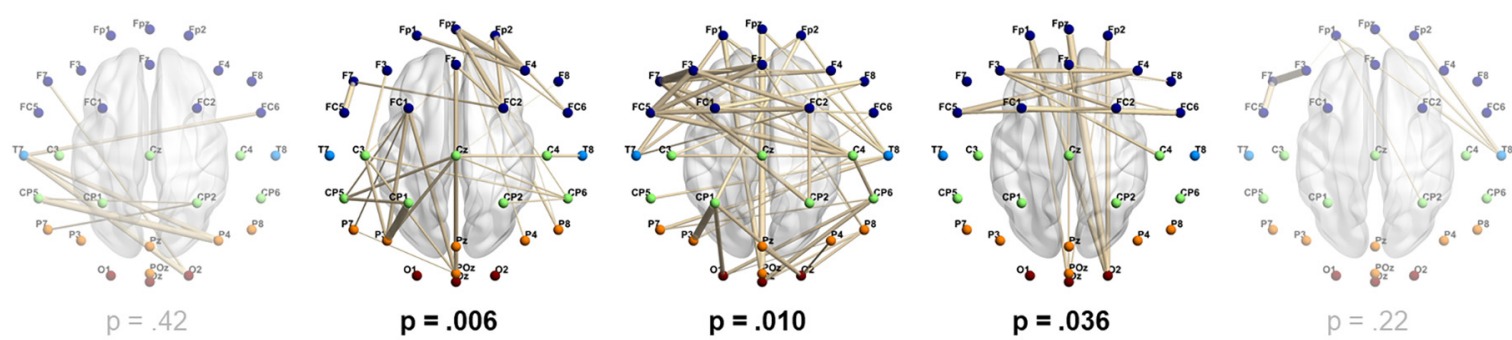

UDE
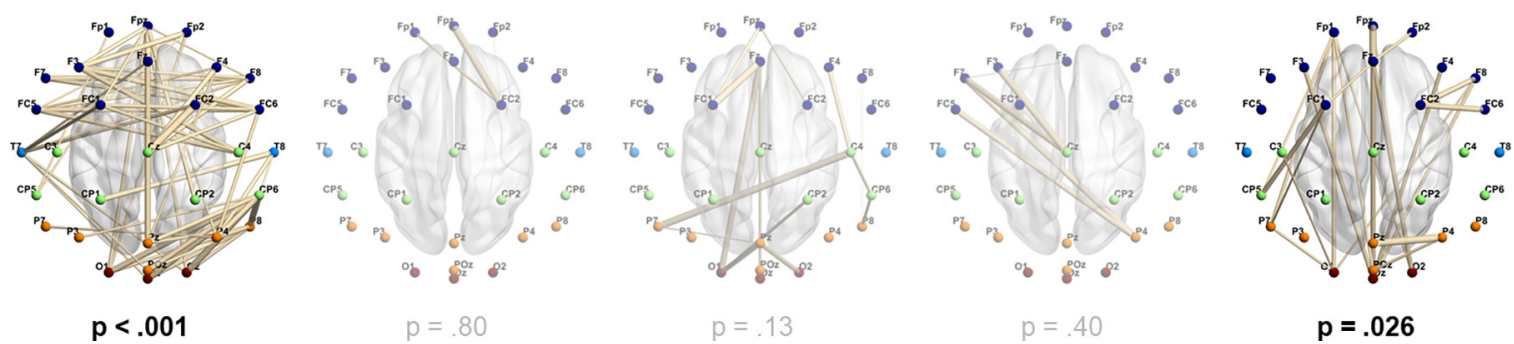

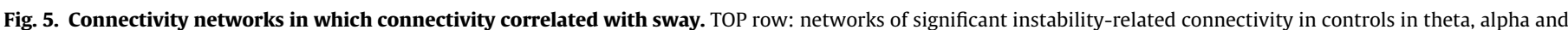

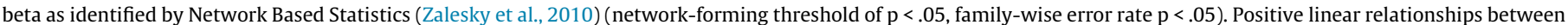

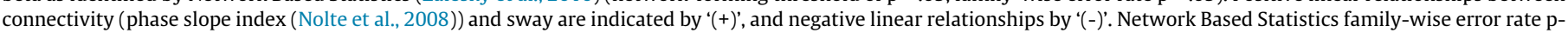

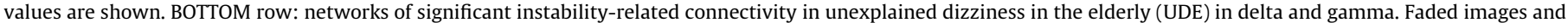
p-values are the best (lowest p-value) corresponding non-significant networks in the other participant group.
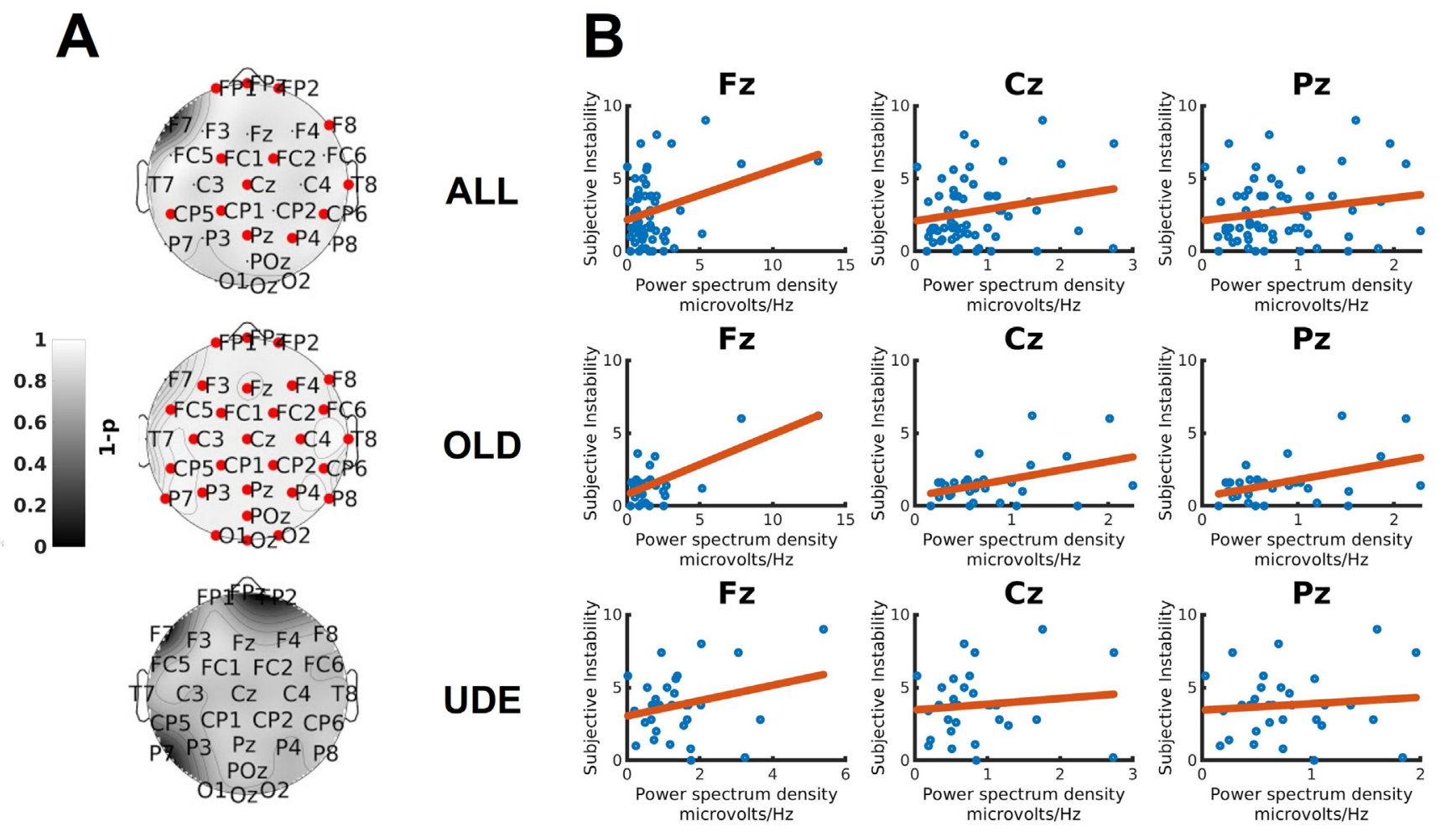

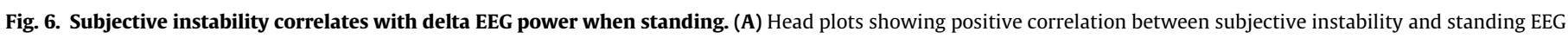

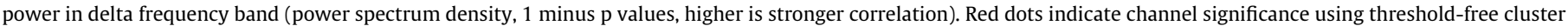

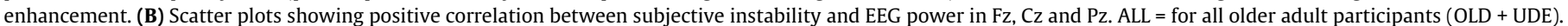
$\mathrm{OLD}=$ older adult controls. UDE = unexplained dizziness in the elderly.

between subjective instability and upright delta power suggests postural symptoms in older adults reflect an awareness of these ageing and small vessel disease-related compensatory neural mechanisms.
We applied a novel approach to postural EEG in continuous balance, applying linear regression to estimate the specific contribution of spontaneous sway in the prediction of power over time. This allowed the estimation of the scaling of cortical responses to 
instability during quiet stance. The validity and statistical power of our approach is confirmed by the replication, within older adults, of the known tendency for delta and gamma power to increase, and theta, alpha and beta bands to decrease, with postural challenge/instability (Fig. 3C) (Edwards et al., 2018; Hülsdünker et al., 2015; Ozdemir et al., 2018; Sipp et al., 2013; Slobounov et al., 2009).

A study investigated the effects of postural challenge on EEG power with increasing age, revealing greater gamma power increases in older adults, compared to younger adults (Ozdemir et al., 2018). They also reported a tendency for older adults to increase fronto-central delta oscillations more substantially than younger adults when balance is threatened, congruent with our finding that delta power increased during periods of instability (Fig. 3C). Our results build on this work, revealing additional theta and alpha desynchronisation on standing in older, compared to younger adults (Fig. 2). The effects of ageing on postural control are thus evident within the ecological setting of quiet standing and are coherent with the symptomatic setting of upright stance in patients with UDE.

Delta power increased with instability in both UDE patients and older controls (Fig. 3C), consistent with a key role for delta oscillations in the response to instability. The finding in delta, that white matter hyperintensity volume correlated positively with the scaling of power with instability in controls, but negatively in UDE is thus of interest.

In controls, increasing small vessel disease likely drives increases in frontal delta oscillations. We suggest this increase is a neural correlate of top-down control which has been linked to an increase in corrective sub-movements with age (Boisgontier et al., 2013; Reuter-Lorenz and Cappell, 2008). Dizzy patients demonstrated similar frontal delta power to controls (Fig. $3 \mathrm{~A}$ and Fig. 3C), but a negative correlation between white matter hyperintensity volume and power-instability coefficients (the linear relationship between power in the standing condition and sway). This implies a deleterious effect of small vessel disease on neural efficiency that precludes a compensatory increase in delta power in dizzy patients. This interpretation is consistent with brain ageing models which predict 'underactivation' when compensatory limits are reached (Mattay et al., 2006; Reuter-Lorenz and Cappell, 2008; Reuter-Lorenz and Park, 2014).

Congruent with a critical role for delta oscillations during periods of instability, connectivity analysis showed that UDE patients engaged a postural control network in the delta frequency band, not evident in controls (Fig. 5). Connectivity in this network increased as instability increased, compatible with recruitment to meet balance demands. Delta oscillations are known to increase during executive processes and the medial prefrontal cortex has been identified as a putative generator (Alper et al., 2006; Knyazev, 2007; Schürmann et al., 2001). The specific engagement of a delta network in UDE is thus consistent with the engagement of an alternative postural control strategy. We suggest this is driven by reduced neural efficiency in other networks implicated in postural control, secondary to the effects of small vessel disease (Harmony, 2013).

We found subjective instability increased with upright delta power across all older adults, as a potential neural correlate of the percept of instability. Of possible relevance, a study during conventional seated EEG reported delta power in the anterior cingulate and insula correlated with symptom burden in chronically dizzy patients, though they had mixed vestibular findings and diagnoses (Alsalman et al., 2016). Importantly, however, we found no significant correlation between baseline (seated) EEG power and subjective instability, confirming the specificity of our finding to upright balance.
White matter hyperintensity volume was only slightly higher in UDE compared to older controls, and white matter hyperintensity volume had differing effects on power-instability coefficients (the linear relationship between power in the standing condition and sway) between the groups. These findings suggest factors other than white matter hyperintensity volume are relevant to UDE. Indeed, educational attainment and IQ, as relevant to cognitive reserve, mediate the effects of small vessel disease on cognition (Backhouse et al., 2017). White matter integrity, a marker of brain reserve, mediates cognitive outcomes (Dufouil et al., 2003; Ter Telgte et al., 2018; Valdés Hernández et al., 2013). That participants with UDE demonstrated lower premorbid intelligence (Table 1), a greater burden of vascular risk factors and different relationships (compared to controls) between white matter hyperintensity volume, and power-instability coefficients suggests resilience factors may have modified the effects of white matter hyperintensity volume. Other (non-cerebrovascular) neuropathological processes are unlikely to have been relevant as most participants with UDE had symptoms for years prior to presentation and alternative diagnoses, including PPPD, did not emerge during at least 6 months of follow-up (Staab et al., 2017).

A limitation of our approach to relating sway in continuous standing to EEG activity is that the causal effects of sway on EEG activity cannot be differentiated from those of EEG activity on sway. Two considerations suggest our power and connectivity findings are nonetheless best interpreted as being driven by spontaneous sway. Firstly, upright stance represents an unstable equilibrium wherein spontaneous instability is both unpredictable and inevitable (Collins and De Luca, 1993). Consequently, under normal circumstances, postural control is best understood as being continually updated in response to periods of spontaneous instability (Loram et al., 2011). The second consideration is that our power findings were in accord with the predictions of multiple studies investigating the effect of postural perturbation, or the transition from a stable to unstable balance (Fig. 3C) (Edwards et al., 2018; Hülsdünker et al., 2015; Ozdemir et al., 2018; Sipp et al., 2013; Slobounov et al., 2009). Thus, though causal effects of EEG activity on sway may have contributed to our results, these were likely small and less significant with respect to our inferences.

\section{Conclusions}

Our results show that central EEG power in theta and alpha frequencies is relevant to balance control. Compared to young controls, theta and alpha power is lower during standing balance (compared to seated rest) in healthy elderly subjects, which may reflect greater executive and attentional resource demands. Unexplained dizziness in the elderly is characterised by further reduction of theta and alpha power on standing. These findings imply pathological ageing of the cortical control of balance may characterise unexplained dizziness in the elderly. Our results confirm SVD is mechanistically relevant to on-line balance control, the neural effects of which influence postural symptoms in healthy ageing, and underpin the syndrome of unexplained dizziness in the elderly. Cortical strategies that compensate for the effects of SVD on postural control appear saturated in older patients with dizziness, suggesting a potential neurophysiological basis for this syndrome.

\section{Author contributions}

RTI, PC, QA, DK and AMB conceived the study. RI, PC and JC collected the data. RTI and AD designed the methodology. RTI anal- 
ysed the data and wrote the first draft of the manuscript. RTI, PC, JC, AE, OG, QA, LM, DK and AMB interpreted the data and critically revised the manuscript. All authors provided approval of the manuscript.

\section{Declaration of Competing Interest}

The authors declare that they have no known competing financial interests or personal relationships that could have appeared to influence the work reported in this paper.

\section{Acknowledgments}

The authors would like to thank Mr. David Buckwell for technical support. The authors would also like to thank Prof L Middleton's team at the Ageing Epidemiology (AGE) Research Unit at the School of Public Health at Imperial College London for facilitating recruitment. The work was made possible by a research grant (R481/0516) from the Dunhill Medical Trust (to AMB) and a block grant from the National Institute for Health Research Imperial Biomedical Research Centre. RTI was supported by the Dunhill Medical Trust. DK is supported by the National Institute for Health Research University College London Hospitals Biomedical Research Centre and a grant from the Meniere's Society.

\section{References}

Ahmad H, Cerchiai N, Mancuso M, Casani AP, Bronstein AM. Are white matter abnormalities associated with "unexplained dizziness"? J Neurol Sci 2015;358 (1-2):428-31.

Alper KR, John ER, Brodie J, Günther W, Daruwala R, Prichep LS. Correlation of PET and qEEG in normal subjects. Psychiatry Res 2006;146(3):271-82.

Alsalman O, Ost J, Vanspauwen R, Blaivie C, De Ridder D, Vanneste S, Kapoula Z. The Neural Correlates of Chronic Symptoms of Vertigo Proneness in Humans. PLoS One 2016;11(4):e0152309. https://doi.org/10.1371/journal. pone.015230910.1371/iournal.pone.0152309.g00110.1371/iournal pone.0152309.g00210.1371/iournal.pone.0152309.g00310.1371/iournal. pone.0152309.g00410.1371/journal.pone.0152309.g00510.1371/journal. pone.0152309.t00110.1371/journal.pone.0152309.t002.

Backhouse EV, McHutchison CA, Cvoro V, Shenkin SD, Wardlaw JM. Early life risk factors for cerebrovascular disease: A systematic review and meta-analysis. Neurology 2017;88(10):976-84.

Bansal R, Peterson BS. Cluster-level statistical inference in fMRI datasets: The unexpected behavior of random fields in high dimensions. Magn Reson Imaging 2018;49:101-15.

Beckmann CF, Jenkinson M, Smith SM. General multilevel linear modeling for group analysis in FMRI. Neuroimage 2003;20(2):1052-63.

Bigdely-Shamlo N, Mullen T, Kothe C, Su K-M, Robbins KA. The PREP pipeline: standardized preprocessing for large-scale EEG analysis. Front Neuroinform 2015;9:16.

Boisgontier MP, Beets IAM, Duysens J, Nieuwboer A, Krampe RT, Swinnen SP. Agerelated differences in attentional cost associated with postural dual tasks: increased recruitment of generic cognitive resources in older adults. Neurosci Biobehav Rev 2013;37(8):1824-37.

Brignole M, Moya A, de Lange FJ, Deharo J-C, Elliott PM, Fanciulli A, et al. 2018 ESC Guidelines for the diagnosis and management of syncope. Eur Heart J 2018;39:1883-948.

Cabeza R. Hemispheric asymmetry reduction in older adults: the HAROLD model. Psychol Aging 2002;17:85-100.

Calic Z, Nham B, Bradshaw AP, Young AS, Bhaskar S, D'Souza M, Anderson CS, Cappelen-Smith C, Cordato D, Welgampola MS. Separating posterior-circulation stroke from vestibular neuritis with quantitative vestibular testing. Clin Neurophysiol 2020;131(8):2047-55.

Castro P, Kaski D, Schieppati M, Furman M, Arshad Q, Bronstein A. Subjective stability perception is related to postural anxiety in older subjects. Gait Posture 2019;68:538-44.

Cerchiai N, Mancuso M, Navari E, Giannini N, Casani AP. Aging with Cerebral Small Vessel Disease and Dizziness: The Importance of Undiagnosed Peripheral Vestibular Disorders. Front Neurol 2017;8:241.

COLLEDGE NICOLAR, WILSON JANETA, MACINTYRE CECILIACA, MACLENNAN WILLIAMJ. The prevalence and characteristics of dizziness in an elderly community. Age Ageing 1994;23(2):117-20.

Collins JJ, De Luca CJ. Open-loop and closed-loop control of posture: a random-walk analysis of center-of-pressure trajectories. Exp Brain Res 1993;95(2):308-18.

Delorme A, Makeig S. EEGLAB: an open source toolbox for analysis of single-trial EEG dynamics including independent component analysis. J Neurosci Methods 2004;134(1):9-21.
Dufouil C, Alperovitch A, Tzourio C. Influence of education on the relationship between white matter lesions and cognition. Neurology 2003;60(5):831-6.

Edwards AE, Guven O, Furman MD, Arshad Q Bronstein AM. Electroencephalographic Correlates of Continuous Postural Tasks of Increasing Difficulty. Neuroscience 2018;395:35-48.

Fife TD, Baloh RW. Disequilibrium of unknown cause in older people. Ann Neurol 1993;34(5):694-702.

Freedman D, Lane D. A nonstochastic interpretation of reported significance levels. J Bus Econ Stat 1983;1:292-8.

Genovese CR, Lazar NA, Nichols T. Thresholding of statistical maps in functional neuroimaging using the false discovery rate. Neuroimage 2002;15(4):870-8.

Guerrero R, Qin C, Oktay O, Bowles C, Chen L, Joules R, Wolz R, Valdés-Hernández MC, Dickie DA, Wardlaw J, Rueckert D. White matter hyperintensity and stroke lesion segmentation and differentiation using convolutional neural networks. Neuroimage Clin 2018;17:918-34.

Harmony T. The functional significance of delta oscillations in cognitive processing. Front Integr Neurosci 2013;7:83.

Hufschmidt A, Dichgans J, Mauritz K-H, Hufschmidt M. Some methods and parameters of body sway quantification and their neurological applications. Arch Psychiatr Nervenkr 1980;228(2):135-50.

Hülsdünker T, Mierau A, Neeb C, Kleinöder H, Strüder HK. Cortical processes associated with continuous balance control as revealed by EEG spectral power. Neurosci Lett 2015;592:1-5.

Jacobson GP, Newman CW. The development of the Dizziness Handicap Inventory. Arch Otolaryngol Head Neck Surg 1990;116(4):424-7.

Jönsson R, Sixt E, Landahl S, Rosenhall U. Prevalence of dizziness and vertigo in an urban elderly population. J Vestib Res 2004;14:47-52.

Knyazev GG. Motivation, emotion, and their inhibitory control mirrored in brain oscillations. Neurosci Biobehav Rev 2007;31(3):377-95.

Lin C-C, Barker JW, Sparto PJ, Furman JM, Huppert TJ. Functional near-infrared spectroscopy (fNIRS) brain imaging of multi-sensory integration during computerized dynamic posturography in middle-aged and older adults. Exp Brain Res 2017;235(4):1247-56.

Loram ID, Gollee H, Lakie M, Gawthrop PJ. Human control of an inverted pendulum: is continuous control necessary? Is intermittent control effective? Is intermittent control physiological? J Physiol 2011;589:307-24.

MacDougall HG, Weber KP, McGarvie LA, Halmagyi GM, Curthoys IS. The video head impulse test: diagnostic accuracy in peripheral vestibulopathy. Neurology 2009;73(14):1134-41.

Makeig S, Debener S, Onton J, Delorme A. Mining event-related brain dynamics. Trends Cogn Sci 2004;8(5):204-10.

Maruff P, Thomas E, Cysique L, Brew B, Collie A, Snyder P, Pietrzak RH. Validity of the CogState brief battery: relationship to standardized tests and sensitivity to cognitive impairment in mild traumatic brain injury, schizophrenia, and AIDS dementia complex. Arch Clin Neuropsychol 2009;24(2):165-78.

Mattay VS, Fera F, Tessitore A, Hariri AR, Berman KF, Das S, Meyer-Lindenberg A, Goldberg TE, Callicott JH, Weinberger DR. Neurophysiological correlates of agerelated changes in working memory capacity. Neurosci Lett 2006;392(12):32-7.

Mensen A, Khatami R. Advanced EEG analysis using threshold-free clusterenhancement and non-parametric statistics. Neuroimage 2013;67:111-8.

Nelson HE. National Adult Reading Test (NART): For the assessment of premorbid intelligence in patients with dementia: Test manual. Nfer-Nelson 1982.

Nichols TE, Holmes AP. Nonparametric permutation tests for functional neuroimaging: a primer with examples. Hum Brain Mapp 2002;15(1):1-25.

Nóbrega-Sousa P, Gobbi LTB, Orcioli-Silva D, Conceiçãoda NR, Beretta VS, Vitório R. Prefrontal Cortex Activity During Walking: Effects of Aging and Associations With Gait and Executive Function. Neurorehabil Neural Repair 2020;34:91524.

Nolte G, Ziehe A, Nikulin VV, Schlögl A, Krämer N, Brismar T, Müller K-R. Robustly estimating the flow direction of information in complex physical systems. Phys Rev Lett 2008;100(23). https://doi.org/10.1103/PhysRevLett.100.234101.

Oostenveld R, Fries P, Maris E, Schoffelen J-M. FieldTrip: Open source software for advanced analysis of MEG, EEG, and invasive electrophysiological data. Comput Intell Neurosci 2011:2011:1-9.

Ozdemir RA, Contreras-Vidal JL, Paloski WH. Cortical control of upright stance in elderly. Mech Ageing Dev 2018;169:19-31.

Papegaaij S, Taube W, Baudry S, Otten E, Hortobágyi T. Aging causes a reorganization of cortical and spinal control of posture. Front Aging Neurosci 2014;6:28.

Park DC, Reuter-Lorenz P. The adaptive brain: aging and neurocognitive scaffolding. Annu Rev Psychol 2009;60:173-96.

Pion-Tonachini L, Kreutz-Delgado K, Makeig S. ICLabel: An automated electroencephalographic independent component classifier, dataset, and website. Neuroimage 2019;198:181-97.

Prieto TE, Myklebust JB, Hoffmann RG, Lovett EG, Myklebust BM. Measures of postural steadiness: differences between healthy young and elderly adults. IEEE Trans Biomed Eng 1996;43(9):956-66.

Reuter-Lorenz PA, Cappell KA. Neurocognitive Aging and the Compensation Hypothesis. Curr Dir Psychol Sci 2008;17(3):177-82.

Reuter-Lorenz PA, Park DC. How does it STAC up? Revisiting the scaffolding theory of aging and cognition. Neuropsychol Rev 2014;24(3):355-70.

Schmidt P, Gaser C, Arsic M, Buck D, Förschler A, Berthele A, Hoshi M, Ilg R, Schmid VJ, Zimmer C, Hemmer B, Mühlau M. An automated tool for detection of FLAIRhyperintense white-matter lesions in Multiple Sclerosis. Neuroimage 2012;59 (4):3774-83. 
Schürmann M, Başar-Eroglu C, Kolev V, Başar E. Delta responses and cognitive processing: single-trial evaluations of human visual P300. Int J Psychophysiol 2001;39(2-3):229-39.

Sipp AR, Gwin JT, Makeig S, Ferris DP. Loss of balance during balance beam walking elicits a multifocal theta band electrocortical response. J Neurophysiol 2013;110(9):2050-60.

Slobounov S, Cao C, Jaiswal N, Newell KM. Neural basis of postural instability identified by VTC and EEG. Exp Brain Res 2009;199(1):1-16.

SMITH S, NICHOLS T. Threshold-free cluster enhancement: addressing problems of smoothing, threshold dependence and localisation in cluster inference. Neuroimage 2009;44(1):83-98.

St George RJ, Hinder MR, Puri R, Walker E, Callisaya ML. Functional Near-infrared Spectroscopy Reveals the Compensatory Potential of Pre-frontal Cortical Activity for Standing Balance in Young and Older Adults. Neuroscience 2021;452:208-18.

Staab JP, Eckhardt-Henn A, Horii A, Jacob R, Strupp M, Brandt T, Bronstein A. Diagnostic criteria for persistent postural-perceptual dizziness (PPPD): Consensus document of the committee for the Classification of Vestibular Disorders of the Bárány Society. J Vestib Res 2017;27(4):191-208.

ter Telgte A, van Leijsen EMC, Wiegertjes K, Klijn CJM, Tuladhar AM, de Leeuw F-E. Cerebral small vessel disease: from a focal to a global perspective. Nat Rev Neurol 2018;14(7):387-98.
Tinetti ME, Richman D, Powell L. Falls efficacy as a measure of fear of falling. J Gerontol 1990;45(6):P239-43.

Valdés Hernández MDC, Booth T, Murray C, Gow AJ, Penke L, Morris Z, Maniega SM, Royle NA, Aribisala BS, Bastin ME, Starr JM, Deary IJ, Wardlaw JM. Brain white matter damage in aging and cognitive ability in youth and older age. Neurobiol Aging 2013;34(12):2740-7.

Veldsman M, Tai X-Y, Nichols T, Smith S, Peixoto J, Manohar S, Husain M Cerebrovascular risk factors impact frontoparietal network integrity and executive function in healthy ageing. Nat Commun 2020;11(1). https://doi. org/10.1038/s41467-020-18201-5.

Wardlaw JM, Smith C, Dichgans M. Small vessel disease: mechanisms and clinical implications. Lancet Neurol 2019:18(7):684-96.

Wardlaw JM, Smith C, Dichgans M. Mechanisms of sporadic cerebral small vessel disease: insights from neuroimaging. Lancet Neurol 2013;12(5):483-97.

Winkler AM, Ridgway GR, Webster MA, Smith SM, Nichols TE. Permutation inference for the general linear model. Neuroimage 2014;92:381-97.

Yardley L, Masson E, Verschuur C, Haacke N, Luxon L. Symptoms, anxiety and handicap in dizzy patients: development of the vertigo symptom scale. J Psychosom Res 1992;36(8):731-41.

Zalesky A, Fornito A, Bullmore ET. Network-based statistic: identifying differences in brain networks. Neuroimage 2010;53(4):1197-207. 